\title{
The Medial Orbitofrontal Cortex-Basolateral Amygdala Circuit Regulates the Influence of Reward Cues on Adaptive Behavior and Choice
}

\author{
Nina T. Lichtenberg, ${ }^{1}$ Linnea Sepe-Forrest, ${ }^{1}$ Zachary T. Pennington, ${ }^{1}$ Alexander C. Lamparelli, ${ }^{1}$ \\ Venuz Y. Greenfield, ${ }^{1}$ and Kate M. Wassum ${ }^{1,2,3,4}$ \\ ${ }^{1}$ Department of Psychology, University of California, Los Angeles, Los Angeles, California 90095, ${ }^{2}$ Brain Research Institute, University of California, \\ Los Angeles, Los Angeles, California 90095, ${ }^{3}$ Integrative Center for Learning and Memory, University of California, Los Angeles, Los Angeles, \\ California 90095, and ${ }^{4}$ Integrative Center for Addictive Disorders, University of California, Los Angeles, Los Angeles, California 90095
}

Adaptive reward-related decision making requires accurate prospective consideration of the specific outcome of each option and its current desirability. Often this information must be inferred based on the presence of predictive environmental events. The basolateral amygdala (BLA) and medial orbitofrontal cortex (mOFC) are two key nodes in the circuitry supporting such outcome expectations, but very little is known about the function of direct connections between these regions. Here, in male rats, we first anatomically confirmed the existence of bidirectional, direct projections between the mOFC and BLA and found that BLA projections to mOFC are largely distinct from those to lateral OFC (IOFC). Next, using pathway-specific chemogenetic inhibition and the outcome-selective Pavlovian-to-instrumental transfer and devaluation tests, we interrogated the function of the bidirectional mOFC-BLA connections in reward-directed behavior. We found evidence that the mOFC $\rightarrow$ BLA pathway mediates the use of environmental cues to understand which specific reward is predicted, information needed to infer which action to choose, and how desirable that reward is to ensure adaptive responses to the cue. By contrast, the BLA $\rightarrow$ mOFC pathway is not needed to use the identity of an expected reward to guide choice but does mediate adaptive responses to cues based on the current desirability of the reward they predict. These functions differ from those we previously identified for the 1OFC-BLA circuit. Collectively, the data reveal the mOFC-BLA circuit as critical for the cue-dependent reward outcome expectations that influence adaptive behavior and decision making.

Key words: appetitive behavior; decision making; devalaution; memory; Pavlovian conditioning; Pavlovian-to-instrumental transfer

\section{Significance Statement}

To make good decisions we evaluate how advantageous a particular course of action would be. This requires understanding what rewarding outcomes can be expected and how desirable they currently are. Such prospective considerations are critical for adaptive decision making but disrupted in many psychiatric diseases. Here, we reveal that direct connections between the medial orbitofrontal cortex and basolateral amygdala mediate these functions. These findings are especially important in light of evidence of dysfunction in this circuit in substance use disorder and mental illnesses marked by poor decision making.

\footnotetext{
Received Apr. 27, 2021; revised June 21, 2021; accepted July 4, 2021.

Author contributions: N.T.L. and K.M.W. designed research; N.T.L., L.S., A.C.L., and V.Y.G. performed research; N.T.L., L.S., Z.T.P., and K.M.W. analyzed data; N.T.L and K.M.W. wrote the paper.

This work was supported by National Institute of Drug Abuse Grants DA035443 (K.M.W.), F31DA044734 and T32DA024635 (N.T.L.), and T32DA024635 (A.C.L.), and the Staglin Center for Behavior and Brain Sciences. We thank Drs. Alicia Izquierdo, Melissa Sharpe, and Melissa Malvaez for feedback.

The authors declare no competing financial interests.

Correspondence should be addressed to Kate M. Wassum at kwassum@ucla.edu.

https://doi.org/10.1523/JNEUROSCI.0901-21.2021

Copyright $\odot 2021$ the authors
}

\section{Introduction}

To make good decisions we evaluate how advantageous a particular course of action would be, given our circumstances, or state. This includes consideration of what outcomes (e.g., specific rewarding events) might occur as well as the current desirability of those potential outcomes. Often the actual outcome we could receive is not readily apparent in the immediate environment. In these cases, we infer the most advantageous option by mentally representing possible outcomes and the current values of these outcomes. These representations are facilitated by predictive environmental events. Stored stimulus-outcome and action-outcome associative memories enable the outcome expectations that influence decision making (Balleine 
and Dickinson, 1998; Delamater, 2012; Fanselow and Wassum, 2015). For example, a restaurant logo on your favorite food-delivery app lets you know that a specific type of food (e.g., tacos) is available, and based on your current state (e.g., Did you just have Mexican for lunch?), you can determine that food's value and decide whether it is a suitable dinner option. Such prospective considerations are critical for adaptive decision making and are disrupted in many psychiatric diseases; yet, much is unknown of the underlying neural circuits of these considerations.

The orbitofrontal cortex (OFC) and basolateral amygdala (BLA) are two key nodes in the circuitry supporting reward outcome representations and their influence over decision making. Damage to either region causes performance deficits when adaptive behavior requires one to represent possible rewarding events (Hatfield et al., 1996; Gallagher et al., 1999; Blundell et al., 2001; Pickens et al., 2003, 2005; Izquierdo et al., 2004; Corbit and Balleine, 2005; Wellman et al., 2005; Machado and Bachevalier, 2007; Ostlund and Balleine, 2007, 2008; Johnson et al., 2009; West et al., 2011; Rhodes and Murray, 2013; Malvaez et al., 2015; Lichtenberg and Wassum, 2017; Panayi and Killcross, 2018). The lateral OFC (lOFC) has been particularly implicated, but the anatomically and functionally distinct medial OFC (mOFC; Wallis, 2011; Izquierdo, 2017; Woon et al., 2020) also participates in appetitive decision making (Noonan et al., 2010, 2017; Stopper et al., 2014; Bradfield et al., 2015, 2018; Dalton et al., 2016; Münster and Hauber, 2018; Malvaez et al., 2018a), especially when the value of rewarding options must be mentally simulated (Bray et al., 2010; Bradfield et al., 2015) and/or compared (Noonan et al., 2010; Rudebeck and Murray, 2011; Stopper et al., 2014; Gourley et al., 2016; Yamada et al., 2018). The mOFC both sends and receives direct excitatory projections to and from the BLA, positioning this as a potential crucial circuit for the influence of reward representations over decision making and adaptive behavior. But little is known of the function of direct $\mathrm{mOFC} \rightarrow \mathrm{BLA}$ projections and nothing of $\mathrm{BLA} \rightarrow \mathrm{mOFC}$ projections. This is limiting for both a basic understanding of appetitive behavior as well as for the understanding and treatment of the myriad psychiatric illnesses marked by dysfunction in the mOFC-BLA circuit (Tanabe et al., 2009; Goldstein and Volkow, 2011; Hahn et al., 2011; Linke et al., 2012; Sladky et al., 2015; Xie et al., 2021).

To address this gap in knowledge, we used a pathway-specific chemogenetic inhibition approach in male rats to uncover the function of $\mathrm{mOFC} \rightarrow \mathrm{BLA}$ and $\mathrm{BLA} \rightarrow \mathrm{mOFC}$ projections in the prospective consideration of specific predicted rewards and their current desirability required for adaptive reward-related behavior. We used Pavlovian and instrumental conditioning procedures that engender the encoding of two distinct, appetitive, sensoryspecific stimulus-outcome memories and two distinct action-outcome memories. We followed this with an outcome-specific Pavlovian-to-instrumental transfer (PIT) test, which assesses the use of these memories to allow reward cues to influence decision making, and an outcome-selective devaluation test to evaluate the ability to use the value of an anticipated reward to guide adaptive behavior. Procedures were identical to our prior investigation of the function of the 1OFC-BLA circuit (Lichtenberg et al., 2017) to facilitate comparison of function of the pathways between the BLA and IOFC versus mOFC.

\section{Materials and Methods}

Subjects

Male Long Evans rats aged 10-12 weeks at the start of the experiment (Charles River Laboratories) were pair housed before surgery and then subsequently housed individually in a vivarium with regulated temperature $\left(68-79^{\circ} \mathrm{F}\right)$ and humidity $(30-70 \%)$. Rats were provided with filtered tap water ad libitum in the home cage and were maintained on a food-restricted $12-14 \mathrm{~g}$ daily diet (LabDiet) to maintain $\sim 85-90 \%$ freefeeding body weight. Rats were handled for $\sim 3 \mathrm{~d}$ before experiment onset. Separate groups of naive rats were used for each experiment. Experiments were performed during the dark phase of a 12:12 h reverse dark/light cycle (lights off at 7 A.M.). All procedures were conducted in accordance with the National Institutes of Health Guide for the Care and Use of Laboratory Animals and were approved by the University of California, Los Angeles Institutional Animal Care and Use Committee.

\section{Surgery}

Standard surgical procedures, described previously (Malvaez et al., 2015, 2019; Lichtenberg et al., 2017), were used for all surgeries. Rats were anesthetized with isoflurane (4-5\% induction, $1-2 \%$ maintenance), and a nonsteroidal anti-inflammatory agent was administered before and after the operation to minimize pain and discomfort. Rats also received postoperative antibiotics.

\section{Anatomical tracing of $m O F C-B L A$ circuit}

Rats $(N=3)$ were infused unilaterally into the BLA with an anterograde and retrograde viral tracer cocktail. The anterograde adeno-associated virus (AAV) expressing the mCherry fluorophore [AAV8-hsyn-mCherry, $4.6 \times 10^{12}$ viral genomes $(\mathrm{vg}) / \mathrm{ml}$; University of North Carolina Vector Core] was mixed 1:1 with the retrogradely trafficked AAV encoding eGFP (AAVrg-hsyn-EGFP, $7.4 \times 10^{12} \mathrm{vg} / \mathrm{ml}$; Addgene) and infused into the BLA [anteroposterior (AP): $-3.0, \mathrm{ML}: \pm 5.1, \mathrm{DV}:-8.6 \mathrm{~mm}$ from bregma; $0.5 \mu \mathrm{L}$ ] at a rate of $0.1 \mu \mathrm{L} / \mathrm{min}$ using a 33 gauge injector. Injectors were left in place for an additional $10 \mathrm{~min}$ to ensure adequate diffusion and to minimize off-target spread along the injector tract. Tissue was collected 8 weeks postsurgery to ensure anterograde and retrograde transport for robust expression in BLA axons and terminals in the MOFC and BLA-projecting mOFC cell bodies.

Anatomical tracing of BLA projections to $M O F C$ and $l O F C$

Rats $(N=4)$ were infused unilaterally with AlexaFluor-594 conjugated cholera toxin B (CTb; $5 \mu \mathrm{g} / \mu \mathrm{L}$; Life Technologies) into the mOFC (AP $+4.1, \mathrm{ML} \pm 0.7, \mathrm{DV}-5.0 \mathrm{~mm}$ from bregma; $0.4 \mu \mathrm{L}$ ) and AlexaFluor488 conjugated $\mathrm{CTb}$ into the $\mathrm{OFC}(\mathrm{AP}+3.0, \mathrm{ML} \pm 3.2, \mathrm{DV}-5.8 \mathrm{~mm}$; $0.6 \mu \mathrm{L}$ ) at a rate of $0.1 \mu \mathrm{L} / \mathrm{min}$ using a 33 gauge injector. Injectors were left in place for an additional $10 \mathrm{~min}$. Tissue was collected 2 weeks postsurgery.

\section{Chemogenetic inhibition of $m O F C \rightarrow B L A$ projections}

Surgery occurred before onset of behavioral training. Rats $(N=11$, final) were infused bilaterally with AAV expressing the inhibitory designer receptor human M4 muscarinic receptor (hM4Di; AAV8-hSyn-hM4DimCherry, $4.8 \times 10^{12}$ or $3.7 \times 10^{12} \mathrm{vg} / \mathrm{ml}$; Addgene; $\left.0.3 \mu \mathrm{L}\right)$ at a rate of $0.1 \mu \mathrm{L} / \mathrm{min}$ into the mOFC (AP: $+4.1, \mathrm{ML}: \pm 0.5, \mathrm{DV}:-5.2 \mathrm{~mm}$ from bregma) using a 33 gauge injector. Injectors were left in place for an additional $10 \mathrm{~min}$ to ensure adequate diffusion and to minimize off-target spread along the injector tract. Twenty-two gauge stainless-steel guide cannulas were implanted bilaterally above the BLA (AP: -3.0, ML: $\pm 5.1, \mathrm{DV}:-7.0 \mathrm{~mm}$ from bregma). Rats recovered for $\sim 16 \mathrm{~d}$ before the onset of behavioral training. Behavioral testing began $6-7$ weeks following surgery to ensure anterograde transport and robust expression in mOFC axons and terminals in the BLA. One rat from this group became ill during behavioral training and was humanely euthanized before testing.

\section{Chemogenetic inhibition of $B L A \rightarrow m O F C$ projections}

Surgery occurred before onset of behavioral training. Rats $(N=9$, final) were infused bilaterally with AAV expressing the inhibitory designer receptor hM4Di (AAV8-hSyn-hM4Di-mCherry, $4.8 \times 10^{12}$ or $3.7 \times 10^{12}$ $\mathrm{vg} / \mathrm{ml}$; Addgene; $0.4 \mu \mathrm{L}$ ) at a rate of $0.1 \mu \mathrm{L} / \mathrm{min}$ into the BLA (AP: -3.0 , ML: $\pm 5.1, \mathrm{DV}:-8.6 \mathrm{~mm}$ from bregma) using a 33 gauge injector. Injectors were left in place for an additional $10 \mathrm{~min}$. Twenty-three gauge stainless-steel guide cannulas were implanted bilaterally above the mOFC (AP: +4.1, ML: $\pm 0.7, \mathrm{DV}:-2.8 \mathrm{~mm}$ from dura). Rats recovered for $\sim 16 \mathrm{~d}$ before the onset of behavioral training. Behavioral testing 
began 6-7 weeks following surgery to ensure anterograde transport and robust expression in BLA axons and terminals in the mOFC.

\section{Behavioral procedures}

Apparatus. Training took place in conditioning chambers (Med Associates) housed within sound- and light-attenuated boxes, described previously (Malvaez et al., 2015, 2019; Lichtenberg and Wassum, 2017; Wassum et al., 2016; Lichtenberg et al., 2017; Collins et al., 2019). Each chamber contained two retractable levers that could be inserted to the left and right of a recessed food-delivery port in the front wall. A photobeam entry detector was positioned at the entry to the food port. Each chamber was equipped with a syringe pump to deliver $20 \%$ sucrose solution in $0.1 \mathrm{ml}$ increments through a stainless-steel tube into one well of the food port and a pellet dispenser to deliver single $45 \mathrm{mg}$ grain food pellets (Bio-Serv) into another well of the same food port. Both a tone and white noise generator were attached to individual speakers on the wall opposite the levers and food port. A $3 \mathrm{~W}, 24 \mathrm{~V}$ house light mounted on the top of the back wall opposite the food-delivery port provided illumination, and a fan mounted on the outer chamber provided ventilation and external noise reduction. Behavioral procedures were similar to those we described previously (Malvaez et al., 2015; Lichtenberg and Wassum, 2017; Lichtenberg et al., 2017).

Pavlovian conditioning. Rats first received eight sessions of Pavlovian training (one session/day) to learn to associate each of two auditory conditional stimuli (CS; $80-82 \mathrm{~dB}, 2 \mathrm{~min}$ duration), tone $(1.5 \mathrm{kHz})$ or white noise, with a specific food reward, sucrose $(20 \%, 0.1$ $\mathrm{ml} /$ delivery) or grain pellets (one $45 \mathrm{mg}$ pellet/delivery; Bio-Serv). CSreward pairings were counterbalanced at the start of each experiment. For half the subjects, tone was paired with sucrose and noise with pellets, with the other half receiving the opposite arrangement. Each session consisted of eight tone and eight white noise presentations. During each 2 min CS the associated reward was delivered on a $30 \mathrm{~s}$ random-time schedule, resulting in an average of four CS-reward pairings per trial. CSs were delivered in pseudorandom order with a variable $2-4 \mathrm{~min}$ (mean $=3 \mathrm{~min}$ ) intertrial interval.

Instrumental conditioning. Rats were then given $11 \mathrm{~d}$ minimum of instrumental training. They received two separate training sessions per day, one with the left lever and one with the right lever, separated by at least $1 \mathrm{~h}$. Each action was reinforced with a different outcome (e.g., left press $\rightarrow$ pellets; right press $\rightarrow$ sucrose; counterbalanced with respect to the Pavlovian contingencies). Each session terminated after 30 outcomes had been earned or $30 \mathrm{~min}$ had elapsed. The reinforcement schedule was escalated ultimately to random ratio (RR)-20 (on average 20 presses required to obtain reward delivery). Rats received 1 training day (minimum) in which their actions were continuously reinforced, then a minimum of 2 training days on RR-2, 2 d on RR-5, and $3 \mathrm{~d}$ on RR-10, before being moved to the final RR-20 schedule for at least $3 \mathrm{~d}$. To be escalated to the next schedule, rats had to earn at least 24 outcomes within $30 \mathrm{~min}$. This requirement had to be met on the final RR-20 schedule for at least 2 training days before subjects were advanced to testing. The maximum number of days required to reach criterion for instrumental acquisition was 15 .

Outcome-selective Pavlovian-to-instrumental transfer test. Following Pavlovian and instrumental conditioning, rats received a set of outcomeselective PIT tests. On the day before each PIT test, rats were given a single 30 min extinction session during which both levers were available, but pressing was not reinforced to establish a low level of responding. During the PIT test, both levers were continuously present, but pressing was not reinforced. After $5 \mathrm{~min}$ of lever-pressing extinction, each $2 \mathrm{~min}$ CS was presented separately four times in pseudorandom order, separated by a fixed $4 \mathrm{~min}$ intertrial interval. No rewards were delivered during CS presentation. Rats received two of each test, one following infusion of vehicle and one following infusion of Clozapine- $n$-oxide $(\mathrm{CNO})$, counterbalanced for order. Rats were given two retraining sessions for each instrumental association (two sessions/day for $2 \mathrm{~d}$ ) and one Pavlovian retraining session in between tests.

Outcome-selective devaluation test. Rats were given a set of two outcome-specific devaluation tests. Immediately before each test, rats were given $1 \mathrm{~h}$ of unlimited access to either the sucrose solution or food pellets in preexposed feeding chambers to establish a sensory-specific satiety and thus selectively devalue the prefed reward. The test consisted of two phases. In the first, both levers were available, and nonreinforced lever pressing was assessed for $5 \mathrm{~min}$. The levers were then retracted, which started the second Pavlovian test phase in which each 2 min CS was presented, without accompanying reward, separately 2 times each in alternating order and separated by a fixed 4 min intertrial interval. Rats received two of each test, one in which food pellets were devalued and one in which the sucrose solution was devalued. After prefeeding and immediately before the test, rats were infused with either vehicle or CNO. Test order and the drug/prefed outcome relationship were counterbalanced across subjects and with respect to the CS-reward relationships. For both the $\mathrm{mOFC} \rightarrow \mathrm{BLA}$ and $\mathrm{BLA} \rightarrow \mathrm{mOFC}$ groups, there were no significant differences in the amount consumed during prefeeding between the future drug conditions ( $\mathrm{mOFC} \rightarrow \mathrm{BLA}$, vehicle: 21.14 grams \pm 2.60 SEM; CNO: $22.35 \pm 3.06 ; t_{(9)}=0.24, p=0.81$; BLA $\rightarrow$ mOFC, vehicle: $19.02 \pm 2.28$; CNO: $\left.16.58 \pm 2.96 ; t_{(8)}=0.58, p=0.57\right)$. Successful devaluation was confirmed by post-test consumption of each food reward. For both groups, the mOFC $\rightarrow$ BLA [vehicle, valued: 8.26 grams \pm 1.35 SEM, devalued: $0.92 \pm 0.31$; CNO, valued: $7.45 \pm 1.26$, devalued: $1.81 \pm 0.78$; outcome (valued v. devalued): $F_{(1,9)}=76.06, p<0.0001$; drug: $F_{(1,9)}=0.0009, p=0.98$; drug $\times$ outcome: $\left.F_{(1,9)}=0.47, p=0.51\right]$ and $\mathrm{BLA} \rightarrow \mathrm{mOFC}$ (vehicle, valued: $4.81 \pm 0.75$, devalued: $3.82 \pm 1.24$; CNO, valued: $9.36 \pm 2.07$, devalued: $1.49 \pm 0.56$; outcome: $F_{(1,8)}=17.86$, $p=0.003$; drug: $F_{(1,8)}=3.21, p=0.11$; drug $\times$ outcome: $F_{(1,8)}=3.95, p=$ 0.08 ), across both drug conditions, rats ate significantly less of the devalued food than the valued food. We excluded $1 \mathrm{mOFC} \rightarrow$ BLA rat from the devaluation test dataset because it did not eat during one of the prefeeding sessions. Rats were given $1-2 \mathrm{~d}$ without behavioral training or testing after each devaluation test to reestablish hunger and then received two retraining sessions for each instrumental association (two sessions/day for $2 \mathrm{~d}$ ) and one Pavlovian retraining session in between tests.

Data collection. Lever presses and/or discrete entries into the fooddelivery port were recorded continuously for each session. For the Pavlovian training and test sessions, the 2 min periods before each CS (preCS) onset served as the baseline for comparison of CS-induced elevations in lever pressing and/or food-port entries.

\section{Chemogenetic inhibition of $m O F C \rightarrow B L A$ projections}

In the $\mathrm{mOFC} \rightarrow \mathrm{BLA}$ group, chemogenetic inhibition was used to inactivate $\mathrm{hM} 4 \mathrm{Di}$-expressing mOFC axons and terminals in the BLA before one PIT test and after prefeeding before one of the devaluation tests. We selected chemogenetic inhibition to allow inhibition throughout the duration of each test. CNO (Tocris Bioscience) was dissolved in artificial cerebral spinal fluid (aCSF) to $1 \mathrm{~mm}$, and $0.5 \mu \mathrm{L}$ was intracranially infused over $1 \mathrm{~min}$ bilaterally into the BLA as previously described (Lichtenberg et al., 2017; Malvaez et al., 2019). Injectors were left in place for at least 1 additional minute to allow for drug diffusion. Testing commenced within 5-10 min following infusion. Using these procedures, we have previously demonstrated effective inactivation of OFC axons and terminals in the BLA both in vivo and ex vivo (Lichtenberg et al., 2017; Malvaez et al., 2019). We have also demonstrated that this dose of CNO when infused into the BLA has no effect on reward-related behavior, BLA activity, or OFC terminal activity in the BLA in the absence of the hM4Di transgene (Lichtenberg et al., 2017; Malvaez et al., 2019). Thus, for the control condition, aCSF was infused into the BLA using identical procedures.

\section{Chemogenetic inhibition of $B L A \rightarrow m O F C$ projections}

For the BLA $\rightarrow$ mOFC group, chemogenetic inhibition was used to inactivate hM4Di-expressing BLA axons and terminals in the mOFC before one PIT test and after prefeeding before one of the devaluation tests. Procedures were identical to those above with the exception that $\mathrm{CNO}$ or aCSF was infused bilaterally into the mOFC $(0.3 \mu \mathrm{L})$. We have previously shown these procedures to effectively attenuate the activity of BLA terminals in the OFC and have found that intra-OFC infusion of this dose of CNO does not affect reward-related behavior, OFC activity, or BLA terminal activity in the OFC in the absence of hM4Di (Lichtenberg 
et al., 2017). Three rats were excluded from this group because of cannula clog and thus the inability to infuse CNO.

\section{Histology}

Rats were deeply anesthetized with Nembutal and transcardially perfused with PBS followed by $4 \%$ paraformaldehyde. Brains were removed and postfixed in $4 \%$ paraformaldehyde overnight, placed into $30 \%$ sucrose solution, then sectioned into $30-40 \mu \mathrm{m}$ slices using a cryostat and stored in PBS or cryoprotectant. For the tracing experiments, fluorescent microscopy was used to confirm expression of eYFP in BLA-projecting mOFC cell bodies. Freefloating coronal sections were mounted onto slides and coverslipped with ProLong Gold mounting medium with DAPI (Invitrogen). The signal for BLA axonal expression of mCherry in the $\mathrm{mOFC}$ was immunohistochemically amplified using antibodies directed against mCherry. Floating coronal sections were washed two times in $1 \times$ PBS for $10 \mathrm{~min}$ and then blocked in a solution of $5 \%$ normal goat serum (NGS) and 1\% Triton X-100 dissolved in PBS for $1-2 \mathrm{~h}$ at room temperature. Sections were then washed three times in PBS for 15 min and then incubated in blocking solution containing rabbit anti-DsRed antibody (1:1000; EMD Millipore) with gentle agitation at $4^{\circ} \mathrm{C}$ for $18-22 \mathrm{~h}$. Florescence imaging was used to visualize AlexaFluor-488 and 594-conjugated $\mathrm{CTb}$ in $\mathrm{OOFC}$ - and mOFC-projecting BLA cells.

To visualize hM4Di-mCherry expression in BLA or mOFC cell bodies, free-floating coronal sections were mounted onto slides and coverslipped with ProLong Gold mounting medium with DAPI (Invitrogen). Axonal expression of hM4Di-mCherry in terminal regions was immunohistochemically amplified. Floating coronal sections were washed two times in $1 \times$ PBS for $10 \mathrm{~min}$ and then blocked in a solution of $5 \%$ NGS and $1 \%$ Triton X-100 dissolved in PBS for 1-2 $\mathrm{h}$ at room temperature. Sections were then washed three times in PBS for $15 \mathrm{~min}$ and then incubated in blocking solution containing rabbit anti-DsRed antibody (1:1000; EMD Millipore) with gentle agitation at $4^{\circ} \mathrm{C}$ for $18-22 \mathrm{~h}$. On the second day, sections were rinsed three times in the blocking solution and incubated in Alexa Fluor 594-conjugated (red) goat secondary antibody (1:500; Invitrogen) for $2 \mathrm{~h}$.

Images were acquired using a Keyence BZ-X710 microscope with a $4 \times, 10 \times$, and $20 \times$ objective (CFI Plan Apo), CCD camera, and BZ-X Analyzer software. Subjects with off-target viral and/or cannula placements were removed from the dataset $(\mathrm{mOFC} \rightarrow \mathrm{BLA}, N=7$; $\mathrm{BLA} \rightarrow \mathrm{mOFC}, N=7$ ). One subject from the $\mathrm{mOFC} \rightarrow \mathrm{BLA}$ group had extensive tissue damage and was also removed. We also excluded $N=2$ from the $\mathrm{mOFC} \rightarrow \mathrm{BLA}$ group and $N=1$ from the $\mathrm{BLA} \rightarrow \mathrm{mOFC}$ group because we were unable to verify expression.

\section{Data analysis}

Behavioral analysis. Behavioral data were processed with Microsoft Excel. Left and/or right lever presses and/or entries into the food delivery port were collected continuously for each training and test session. For the last day of Pavlovian training, we compared the rate of food-port entries between the CS-probe (after CS onset, before reward delivery) and the preCS baseline periods. Data were averaged across trials for each CS and then averaged across the two CSs. Press rates on the last day of instrumental training were averaged across levers. For the PIT test, the baseline lever-press rate (presses/min) averaged across both levers during the 2 min periods immediately before the onset of each CS was compared with that during the CS periods. During the CS periods, lever pressing was separated for presses on the lever that during training, earned the same outcome as the presented cue (CS-Same presses) versus those on the other available lever (CS-Different presses). Data were averaged across trials for each CS and then averaged across the two CSs. Rate of entries into the food delivery port were also compared between the baseline and CS periods, averaged across both CSs. For the devaluation test instrumental phase, the lever-press rate was compared between the lever that in training earned the prefed food outcome (i.e., the devalued outcome) and that which had previously earned the nonprefed (valued) outcome. During the Pavlovian phase the rate of entries into the fooddelivery port were compared between the $2 \mathrm{~min}$ preCS baseline periods and the CS periods, which were separated by the CS that predicted the devalued outcome versus the CS that predicted the valued outcome.

Image Analysis. For the $\mathrm{CTb}$ tracing experiment, for each subject we analyzed two slices each of the anterior $(-2.3$ to $-2.56 \mathrm{~mm}$ posterior to bregma), middle ( -2.8 to $-3.14 \mathrm{~mm}$ posterior to bregma), and posterior $(-3.3$ to -3.6$)$ BLA. We used $20 \times$ images from the center of the lateral amygdala (LA) and BLA for each analysis. Single- and double-labeled neurons for each fluorophore were counted using a custom-written script in ImageJ (version 1.50i; National Institutes of Health). Cells counts were totaled across the LA and BLA and across slices.

Statistical analysis. Datasets were analyzed by two-tailed, paired Student's $t$ tests, one- or two-way repeated-measures ANOVA, as appropriate (GraphPad Prism, GraphPad; IBM SPSS). post hoc tests used the Bonferroni correction. All data were tested for normality before analysis with ANOVA, and the Greenhouse-Geisser correction was applied to mitigate the influence of unequal variance between conditions if the assumption of sphericity was not met. Alpha levels were set at $p<0.05$.

\section{Rigor and reproducibility}

Group sizes were estimated a priori based on prior work using male Long Evans rats in this behavioral task (Malvaez et al., 2015; Lichtenberg and Wassum, 2017; Lichtenberg et al., 2017) and to ensure counterbalancing of CS-reward and lever-reward pairings. Investigators were not blinded to condition because they were required to administer drugs. All behaviors were scored using automated software (MedPC). Each behavioral experiment included at least one replication cohort, and cohorts were balanced by viral group, CS-reward and lever-reward pairings, and drug test order, before the start of the experiment.

\section{Data and Code Availability}

All data supporting the findings of this study are available from the corresponding author on request.

\section{Results}

\section{The BLA and mOFC are bidirectionally connected}

Using anterograde and retrograde viral-mediated tracing, we first confirmed the existence of direct connections between the BLA and mOFC, as identified previously (Kita and Kitai, 1990; Hoover and Vertes, 2011; Heilbronner et al., 2016; Reppucci and Petrovich, 2016; Malvaez et al., 2019; Barreiros et al., 2021). An anterograde and retrograde tracer was infused into the BLA of male rats (Fig. $1 A ; N=3$ ). We detected robust expression of the retrograde tracer fluorophore in BLA-projecting $\mathrm{mOFC}$ cell bodies, confirming a direct $\mathrm{mOFC} \rightarrow \mathrm{BLA}$ projection. We also detected robust axonal expression of the anterograde tracer fluorophore in the $\mathrm{mOFC}$, indicating the presence of $\mathrm{BLA} \rightarrow \mathrm{mOFC}$ projections. Thus, the $\mathrm{MOFC}$ and BLA share direct, bidirectional connections.

The BLA also projects to the lOFC (Morecraft et al., 1992; Lichtenberg et al., 2017; Barreiros et al., 2021), and we previously found that activity in these projections participates in decision making and adaptive behavior (Lichtenberg et al., 2017). Thus, we next asked whether the BLA pathway to the $\mathrm{mOFC}$ is distinct or overlapping with that to the IOFC, that is, whether the BLA sends collateral projections to both mOFC and lOFC. We injected retrograde tracers into the $\mathrm{OOFC}$ and $\mathrm{mOFC}$ of male rats (Fig. $1 B-C ; N=4$ ). We detected expression of both tracer fluorophores in BLA cell bodies across the AP extent of the BLA, confirming the presence of BLA projections to both $1 O F C$ and mOFC (Fig. $1 D$; lateral amygdala, $\mathrm{AP} \times$ projection: $F_{(10,30)}=$ $4.95, p=0.0003$; AP: $F_{(5,15)}=4.10, p=0.02$; projection: $F_{(2,6)}=$ 8.23, $p=0.02$; BLA, AP $\times$ projection: $F_{(10,30)}=4.21, p=0.001$; AP: $F_{(5,15)}=1.31, p=0.31$; projection: $\left.F_{(2,6)}=8.78, p=0.001\right)$. lOFC-projecting cells were more prominent than $\mathrm{mOFC}$ projectors in the anterior BLA. We detected more labeling of 

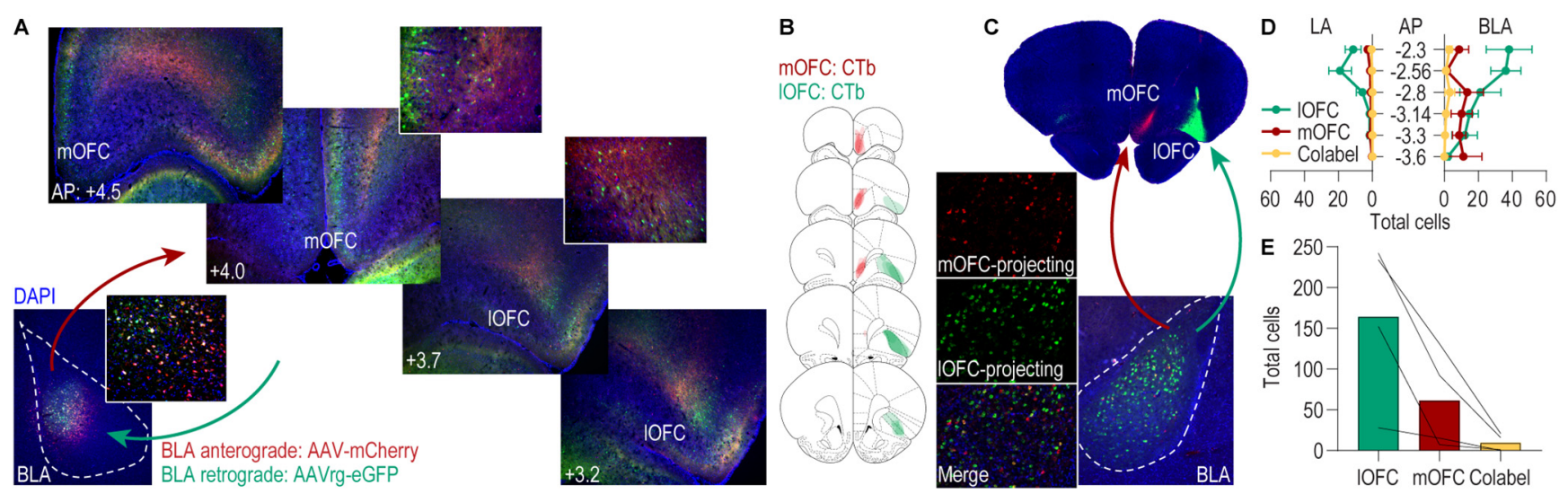

Figure 1. The BLA and mOFC are bidirectionally connected. $A$, Representative images of the anterograde (mCherry) and retrograde (eGFP) tracer expression in the BLA infusion site (bottom left) and of BLA axons and terminals (mCherry) and BLA-projecting cell bodies (eGFP) throughout the medial and lateral OFC. Insets, A 20× image of cell body and axonal expression. $\boldsymbol{B}$, Schematic representation of the spread of the retrograde tracers AlexaFluor-594 (Tb (red, $\mathrm{mOFC}$ ) and AlexaFluor-488 (Tb (green, IOFC) in the OFC infusion sites. C, Representative images of Alexafluor-594 CTb and AlexaFluor-488 CTb spread in the mOFC and IOFC infusion sides, and of mOFC- and IOFC-projecting cell bodies in the BLA. D, Quantification of AlexaFluor-594 CTb (mOFC projecting), AlexaFluor-488 (Tb-labeled (IOFC projecting), and colabeled cells across the AP axis of the LA and BLA. E, Quantification of AlexaFluor-594 CTb (m0FC projecting), AlexaFluor-488 CTb-labeled (IOFC projecting), and co-labeled cells totaled across LA and BLA for all slices.

$\mathrm{BLA} \rightarrow \mathrm{lOFC}$ projecting cells than $\mathrm{BLA} \rightarrow \mathrm{mOFC}$ projecting cells, likely because of the larger size, and thus tracer infusion volume, of the lOFC relative to mOFC. Critically, we detected very few colabeled cells, indicating that for the most part, the BLA pathways to the mOFC and lOFC are distinct (Fig. $1 E ; F_{(2,6)}=10.11$, $p=0.01$.

The $\mathrm{mOFC} \rightarrow$ BLA pathway regulates the influence of stimulus-outcome memories over decision making and adaptive conditional responding

We next used chemogenetic inhibition to interrogate the function of $\mathrm{mOFC} \rightarrow \mathrm{BLA}$ projections in the influence of stimulusoutcome and action-outcome memories over decision making and adaptive conditional behavior (Fig. $2 A$ ). We expressed the inhibitory designer receptor hM4Di in the $\mathrm{mOFC}$ and placed guide cannula bilaterally over the BLA near hM4Di-expressing mOFC axons and terminals (Fig. 2B,C; $N=11$ ). This allowed us to later infuse $\mathrm{CNO}(1 \mathrm{~mm})$ to inactivate $\mathrm{mOFC}$ axons and terminals in the BLA, as we have previously validated both in vivo and ex vivo (Lichtenberg et al., 2017; Malvaez et al., 2019).

Rats were food deprived and given Pavlovian and instrumental conditioning using procedures that engender the encoding of rich, sensory-specific stimulus-outcome and action-outcome memories (Ostlund and Balleine, 2008; Malvaez et al., 2015; Lichtenberg et al., 2017; Lichtenberg and Wassum, 2017; Sias et al., 2021). For Pavlovian conditioning, each of two 2 min auditory CSs (white noise and tone) were associated with intermittent delivery of one of two distinct food rewards (sucrose solution or food pellets, e.g., white noise-sucrose/tone-pellet). Thus, each cue sets the state in which a specific reward can be expected. During each session, each cue was presented eight times in pseudorandom order (variable average $3 \mathrm{~min}$ intertrial interval) for $2 \mathrm{~min}$, during which its associated reward was delivered on average every $30 \mathrm{~s}$. Evidence of simple Pavlovian conditioning was detected in the goal-approach response (entries into the food delivery port) to the cue before reward delivery (baseline: 8.23 entries/min \pm 0.89 SEM; CS probe period: $18.72 \pm 1.58 ; t_{(10)}=$ $12.57, p<0.0001)$. Rats then received instrumental conditioning to learn that two different actions (left or right lever press) each earned one of the two food rewards (e.g., left press $\rightarrow$ sucrose/ right press $\rightarrow$ pellets), ultimately on a random-ratio 20 schedule of reinforcement, thus establishing two action-outcome memories. Rats reached a final average press rate of $35.18 \pm 3.13$ presses/min.

We first asked whether activity in $\mathrm{mOFC} \rightarrow$ BLA projections is necessary for the influence of stimulus-outcome and actionoutcome memories over decision making using the outcomespecific PIT test. Rats received two PIT tests, counterbalanced for order, one following intra-BLA infusion of CNO to inactivate hM4Di-expressing mOFC axons and terminals in the BLA and one following infusion of aCSF vehicle. We chose a vehicleinfused control to provide a within-subject comparison, based on evidence that CNO when infused at this dose into the BLA has no effect on the expression of PIT, similar reward-related behaviors, BLA activity, or OFC terminal activity in the BLA in the absence of the hM4Di transgene (Lichtenberg et al., 2017; Malvaez et al., 2019). During each test, both levers were continuously present, but lever pressing was not rewarded. Each CS was presented four times (also without accompanying reward), with intervening CS-free baseline periods, to assess its influence on action performance and selection in the novel choice scenario. The test was unrewarded to force subjects to rely on memory of the predicted rewards. Because the cues are never associated with the instrumental actions, this test assesses the ability, on cue presentation, to represent the specific predicted reward and use this to motivate choice of the action known to earn the same unique reward (Kruse et al., 1983; Colwill and Motzkin, 1994; Gilroy et al., 2014; Corbit and Balleine, 2016). If subjects are able use both the stimulus-outcome and action-outcome memories to enable accurate reward representation, then cue presentation should cause them to increase their lever presses selectively on the action that earned the same outcome as predicted by that cue. This is consistent with the interpretation that rats use the cues to know which reward is predicted and, thus, infer which action would be most advantageous. Rats showed this outcome-specific PIT effect in the control condition (Fig. 2D). Cue presentation increased presses selectively on the lever that in training earned the same outcome as the presented cue, relative to the lever that earned the different outcome. Conversely, the influence of the cues over lever-press choice was greatly attenuated following intra-BLA $\mathrm{CNO}$ infusion [Fig. $2 D$; drug $\times \mathrm{CS} /$ lever: $F_{(1.4,14)}=4.20, p=$ 0.049; drug (vehicle vs CNO): $F_{(1,10)}=9.51, p=0.01$; CS/lever 


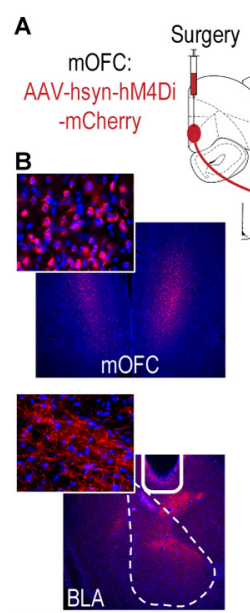

DAPI hM4Di
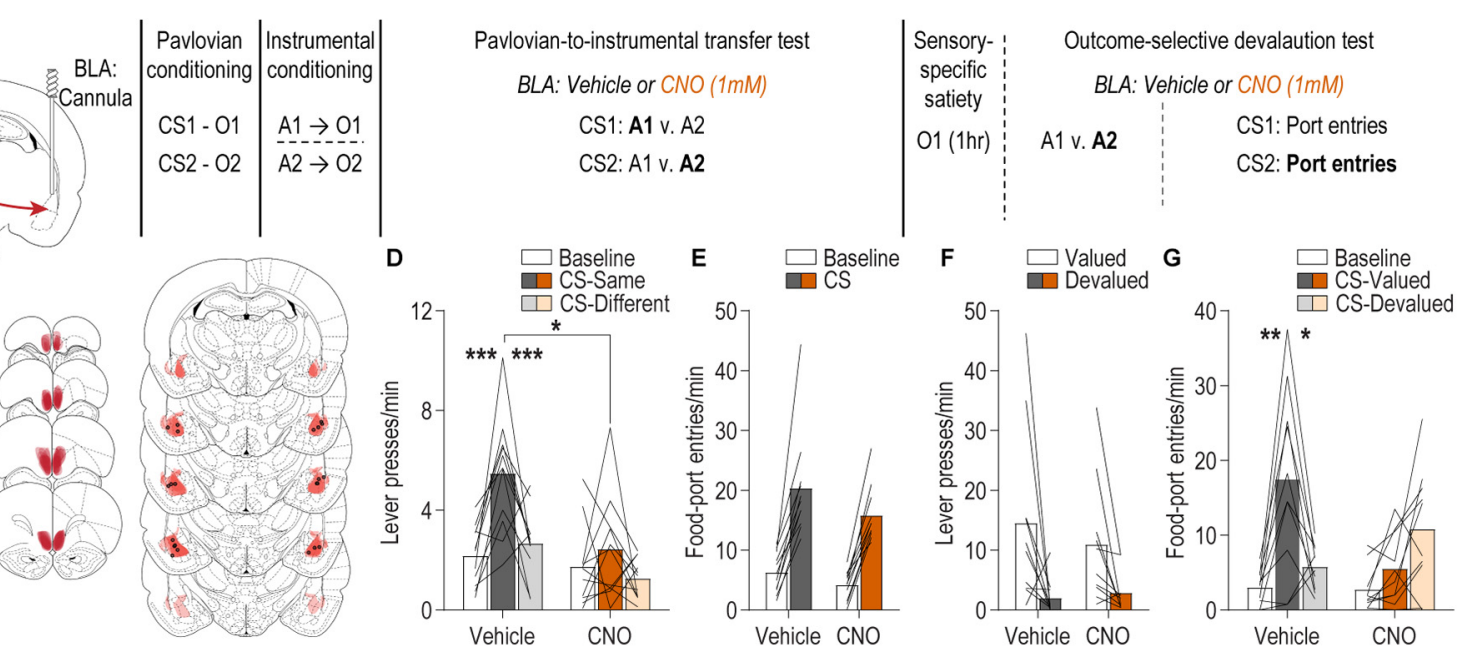

Figure 2. The $\mathrm{mOF} \rightarrow \mathrm{BLA}$ pathway regulates the influence of stimulus-outcome memories over decision making and adaptive conditional responding. $\boldsymbol{A}$, Schematic of behavioral training, testing, and chemogenetic inactivation strategy. CS, conditional stimulus (white noise or tone); 0 , outcome (sucrose solution or food pellet); A, action (left or right lever press). $\boldsymbol{B}$, Representative fluorescent image of hM4Di-mCherry expression in mOFC cell bodies and immunofluorescent image of mOFC axons and terminals in the BLA near the implanted guide cannula. C, Schematic representation of hM4Di-mCherry expression in mOFC and axonal expression and injector tip placements in BLA for all subjects. D, Lever-press rate (lever presses/min) during the PIT test averaged across levers during the preCS baseline periods and during the CS periods on the lever earning the same outcome as the presented CS and the alternate lever (Different). Data are averaged across trials and CSS. E, Rate of entries into the food-delivery port (food-port entries/min) during the PIT test for the preCS baseline periods and during CS presentation (averaged across trials and (Ss). $\boldsymbol{F}$, Average lever-press rate during the instrumental choice phase of the outcome-specific devaluation test. Presses separated for those on the action that in training earned the valued versus devalued (prefed) reward. $\boldsymbol{G}$, Rate of entries into the food-delivery port during the outcome-specific devaluation test, averaged across trials, during the preCS baseline period, and during presentation of the CS predicting the valued and devalued (prefed) rewards. Lines represent individual subjects. ${ }^{*} p<0.05,{ }^{* *} p<0.01,{ }^{* * *} p<0.001$.

(preCS, CS-Same vs CS-Different): $\left.F_{(1.5,14.7)}=10.87, p=0.002\right]$. This effect was selective to cue-influenced decision making. Inactivation of $\mathrm{mOFC} \rightarrow \mathrm{BLA}$ projections neither affected baseline lever-pressing activity $(p>0.9999$, Bonferroni-corrected post hoc comparison), nor conditional approach responses to the shared food-delivery port during the cues [Fig. 2E; CS (baseline vs CS): $F_{(1,10)}=71.66, p<0.0001$; drug: $F_{(1,10)}=4.14, p=0.07$; drug $\left.\times C S: F_{(1,10)}=1.70, p=0.22\right]$. Thus, activity in $\mathrm{mOFC} \rightarrow \mathrm{BLA}$ projections is not needed to support general reward pursuit activity or conditional approach responses but is necessary to use appetitive cues to know which reward is predicted and, thus, bias decision making.

PIT requires that the subjects both know which outcome is predicted by the presented cue and which action earns that same outcome. Thus, the disrupted expression of PIT by inactivation of $\mathrm{mOFC} \rightarrow \mathrm{BLA}$ projections could reflect a function for this pathway in stimulus-outcome memory, action-outcome memory, or both. To arbitrate among these possibilities, we retrained rats on both the Pavlovian and instrumental contingencies and gave them a set of outcome-specific devaluation tests. This also provided an opportunity to assess the contribution of $\mathrm{mOFC} \rightarrow \mathrm{BLA}$ projection activity to the ability to use the value of an anticipated reward to guide adaptive behavior. Before each test, one of the food rewards was devalued by sensory-specific satiety ( $1 \mathrm{~h}$ prefeeding). No manipulation was made during this prefeeding to allow devaluation learning to proceed undisrupted. Immediately following the prefeeding, rats received an infusion of vehicle or CNO into the BLA and were then given a two-phase test. In the first phase, rats received $5 \mathrm{~min}$ access to both levers in a choice. Lever pressing was not reinforced to force subjects to rely on their actionoutcome memories. If rats can use their action-outcome memories to represent the expected outcome of each action and its value, then they should select the action that earns the valued reward, downshifting responding on the action that earns the devalued reward. Rats showed this sensitivity of instrumental choice to outcome-selective devaluation in the control condition and when $\mathrm{mOFC} \rightarrow \mathrm{BLA}$ projections were inactivated [Fig. $2 F$; lever (valued vs devalued): $F_{(1,9)}=15.32, p=0.004$; drug: $F_{(1,9)}=0.20, p=0.67$; drug $\times$ lever: $F_{(1,9)}=0.47, p=$ $0.51)$. Thus, activity in $\mathrm{mOFC} \rightarrow \mathrm{BLA}$ projections is not necessary to retrieve action-outcome memories or use them to guide choice behavior. In the second phase, the levers were retracted, and each cue was presented twice in alternating order. Again, the subjects were forced to rely on memory, in this case the stimulus-outcome memories, because the cues were not accompanied by the associated reward. If rats can use the cues to know which reward is predicted and how valuable that reward currently is, then they should be able to infer that it is advantageous to respond to the cue signaling the valued outcome by checking the food-delivery port, but it is not advantageous to respond during the cue signaling the devalued outcome. This was apparent in the control condition. Following intra-BLA vehicle infusion, rats showed robust conditional food-port approach responses to the cue signaling the valued reward but attenuated responses to the cue signaling the devalued reward. Inactivation of $\mathrm{mOFC} \rightarrow \mathrm{BLA}$ projections prevented subjects from adapting the conditional goal-approach responses based on the value of the predicted reward [Fig. $2 G$; drug $\times$ CS: $F_{(1.3,11.9)}=8.07, p=$ 0.01 ; drug: $F_{(1,9)}=1.32, p=0.28$; CS (valued vs devalued): $\left.F_{(1.5,13.9)}=20.47, p=0.0001\right]$. Thus, activity in $\mathrm{mOFC} \rightarrow \mathrm{BLA}$ projections is necessary for adaptive responses to a cue based on the current value of the predicted reward. Together with the PIT results, this suggests that $\mathrm{mOFC} \rightarrow \mathrm{BLA}$ projection activity mediates the use of environmental cues to represent which specific reward is predicted and the desirability of that reward, both of which are critical for adaptive decision making.

The $\mathrm{BLA} \rightarrow$ mOFC pathway mediates adaptive cue responses based on the value of the predicted reward

We next asked whether BLA projections back to the mOFC are similarly involved appetitive behavior (Fig. $3 A ; N=9$ ). The 

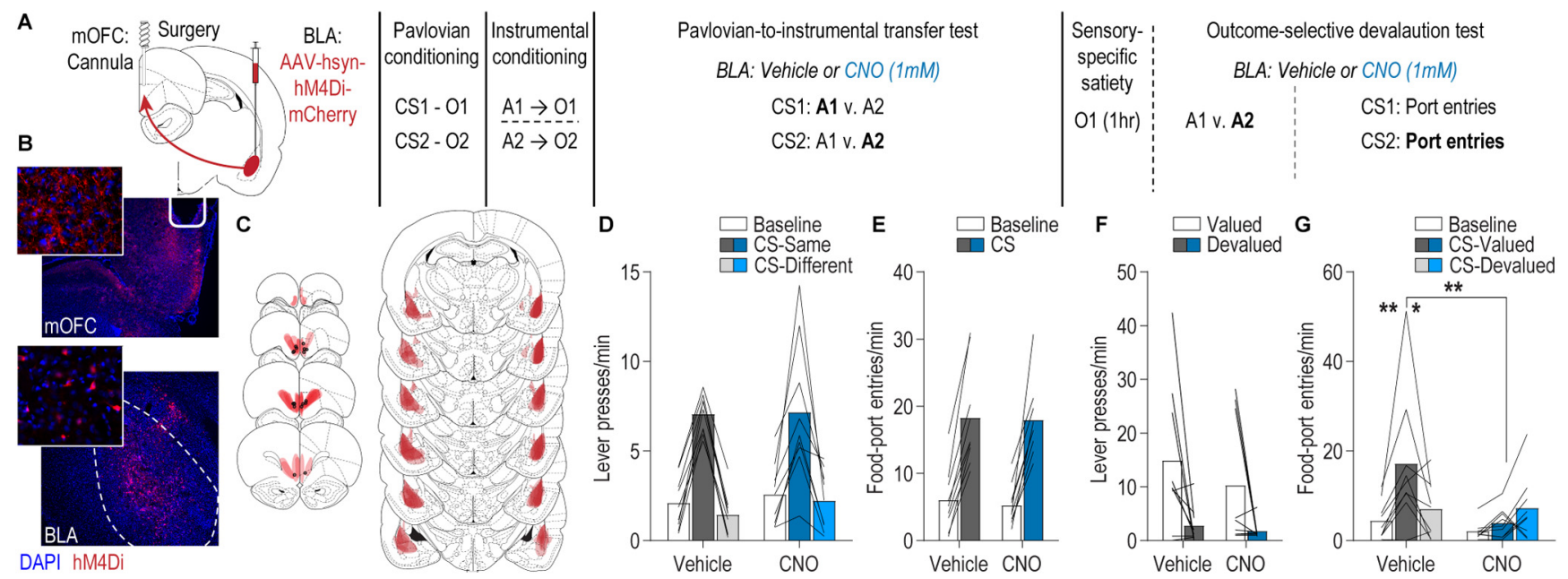

Figure 3. The $B L A \rightarrow \mathrm{mOFC}$ pathway regulates adaptive cue responses based on predicted reward value. $\boldsymbol{A}$, Schematic of behavioral training, testing, and chemogenetic inactivation strategy. CS, conditional stimulus (white noise or tone); 0 , outcome (sucrose solution or food pellet); $A$, action (left or right lever press). $\boldsymbol{B}$, Representative fluorescent image of hM4Di-mCherry expression in cell bodies of the BLA and immunofluorescent image of BLA axons and terminals in the mOFC near the implanted guide cannula. $C$, Schematic representation of hM4Di-mCherry expression in BLA and axonal expression and injector tip placements in mOFC for all subjects. D, Lever-press rate (lever presses/min) during the PIT test averaged across levers during the preCS baseline periods and during the CS periods on the lever earning the same outcome as the presented CS and the alternate lever (Different). Data are averaged across trials and CSs. $\boldsymbol{E}$, Rate of entries into the food-delivery port (food-port entries/min) during the PIT test for the preCS baseline periods and during $C S$ presentation (averaged across trials and $(S S)$. $F$, Average lever-press rate during the instrumental phase of the outcome-specific devaluation test. Presses separated for those on the action that in training earned the valued versus devalued (prefed) reward. $\mathbf{G}$, Rate of entries into the food-delivery port during the outcome-specific devaluation test, averaged across trials, during the preCS baseline period, and during presentation of the $C S$ predicting the valued and devalued (prefed) rewards. Lines represent individual subjects. ${ }^{*} p<0.05,{ }^{* *} p<0.01$.

experiment was identical, except this time we expressed the inhibitory designer receptor hM4Di in the BLA and placed guide cannula bilaterally over the mOFC near hM4Di-expressing BLA axons and terminals (Fig. 3B,C). This allowed a later $\mathrm{CNO}$ ( $1 \mathrm{~mm}$ ) infusion to inactivate BLA axons and terminals in the mOFC as we have validated previously (Lichtenberg et al., 2017). We have also previously shown that in the absence of the hM4Di transgene, $\mathrm{CNO}$ infusion at this dose into the OFC does not affect OFC activity, BLA terminal activity in the OFC, or rewardrelated behavior (Lichtenberg et al., 2017), and thus used a within-subject, vehicle-infused control condition. Rats provided evidence of successful Pavlovian conditioning in their final average goal-approach responses to the cues (baseline: 6.60 entries/min \pm 0.69 SEM; CS probe period: $14.64 \pm$ $\left.1.71 ; t_{(8)}=6.56, p=0.0002\right)$ and also acquired performance of the instrumental actions, reaching a final average press rate of $39.70 \pm 2.51 \mathrm{presses} / \mathrm{min}$.

In contrast to the $\mathrm{mOFC} \rightarrow \mathrm{BLA}$ pathway, activity of $\mathrm{BLA} \rightarrow \mathrm{mOFC}$ projections was found to be unnecessary for the expression of PIT. Following intra-mOFC infusion of either vehicle or $\mathrm{CNO}$, the reward-predictive cues were capable of biasing lever-pressing choices toward the lever that during training earned the same outcome as the presented cue relative to the lever that earned the different outcome (Fig. $3 D$; CS/lever: $F_{(1.5,12)}=63.71, p<0.0001$; drug: $F_{(1,8)}=0.26, p=0.62$; drug $\times$ CS/lever: $F_{(1.2,9.5)}=0.22, p=$ $0.69)$. During this PIT test, the conditional goal-approach response was also similar between groups (Fig. $3 E$; CS: $F_{(1,8)}=83.04, p<0.0001$; drug: $F_{(1,8)}=0.04, p=0.86$; drug $\times$ CS: $\left.F_{(1,8)}=0.06, p=0.81\right)$. Thus, activity in BLA $\rightarrow \mathrm{mOFC}$ projections is neither needed to retrieve action-outcome or stimulus-outcome memories nor to use this information to generate representations of the specific predicted reward to influence decision making.

Activity in BLA $\rightarrow$ mOFC projections was, however, critical to adapt approach responses to the cues based on the value of the predicted reward. During the outcome-specific devaluation test, in both the intra-mOFC vehicle and $\mathrm{CNO}$ conditions, rats showed sensitivity of their instrumental choice behavior to outcome-specific devaluation, downshifting responding on the action that in training earned the devalued reward (Fig. $3 F$; lever: $F_{(1,8)}=20.60, p=0.002$; drug: $F_{(1,8)}=0.57, p=0.47$; drug $\times$ lever: $\left.F_{(1,8)}=0.22, p=0.65\right)$. This confirmed that BLA $\rightarrow$ mOFC projection activity is not needed to retrieve action-outcome memories and further demonstrated that these projections are not needed for general value-based decision making. However, inactivation of $\mathrm{BLA} \rightarrow \mathrm{mOFC}$ projections did prevent subjects from adapting Pavlovian conditional food-port approach responses based on the value of the predicted reward. Following intra-mOFC vehicle infusion, rats showed robust conditional food-port approach responses to the cue signaling the valued reward and attenuated responses to the cue signaling the devalued reward. This sensitivity of the conditional goal-approach response to devaluation was abolished following intra-mOFC CNO infusion [Fig. $3 G$; drug $\times$ CS: $F_{(2,16)}=5.01, p=0.02$; drug: $F_{(1,8)}=2.98, p=0.12$; CS (valued vs devalued): $\left.F_{(2,16)}=9.86, p=0.002\right]$. Thus, based on the PIT results, activity in the $\mathrm{BLA} \rightarrow \mathrm{mOFC}$ pathway is not needed to use the cues to know the identity of predicted reward, but the devaluation results indicate it is required to use the current value of that reward to infer how advantageous it would be to respond to the cue.

\section{Discussion}

Here, we interrogated the function of mOFC-BLA circuitry in the prospective considerations that underlie adaptive rewardrelated behavior. Using anatomical tracing, we confirmed the existence of bidirectional, direct pathways between $\mathrm{mOFC}$ and BLA and found that BLA projections to the mOFC and IOFC are largely distinct. Using pathway-specific chemogenetic inhibition, we found that $\mathrm{mOFC} \rightarrow \mathrm{BLA}$ projection activity is critical for using stimulus-outcome memories to guide decision making 
based on which specific reward is expected and adaptive cue responses based on the desirability of that predicted outcome. $\mathrm{BLA} \rightarrow \mathrm{mOFC}$ projection activity, however, is only needed to adapt cue responses based on the desirability of the predicted reward.

We found that $\mathrm{mOFC} \rightarrow \mathrm{BLA}$ pathway activity is critical for using predictive environmental cues to know which specific reward is predicted and the current value of that option. mOFC neuronal activity can represent a cue-reward memory (Namboodiri et al., 2019) and is necessary, across species, for the use of such memories to inform adaptive behavior (Noonan et al., 2010, 2017; Bradfield et al., 2015, 2018). Our data indicate this function is, at least in part, achieved via direct projections to the BLA. We have previously found that $\mathrm{mOFC} \rightarrow \mathrm{BLA}$ pathway activity mediates the retrieval of the incentive value of an expected food reward to ensure its adaptive pursuit (Malvaez et al., 2019). Incentive value is another form of state-dependent reward memory (food has high value when hungry, but low when sated). Thus, $\mathrm{mOFC} \rightarrow \mathrm{BLA}$ projections may be responsible for using the current state, defined both by external and internal physiological cues, to make a judgment about how advantageous a certain course of action might be.

This function of $\mathrm{mOFC} \rightarrow \mathrm{BLA}$ projections is distinct from that of $\mathrm{lOFC} \rightarrow \mathrm{BLA}$ projections. Using the same procedures, we previously found that $\mathrm{IOFC} \rightarrow \mathrm{BLA}$ projections are not needed for reward cues to bias choice toward the predicted reward (Lichtenberg et al., 2017). They are also unnecessary for retrieving the incentive value of an expected reward (Malvaez et al., 2019). Rather, 1 OFC $\rightarrow$ BLA pathway activity drives the learning of these state-dependent reward memories (Malvaez et al., 2019; Sias et al., 2021), a function that requires the BLA itself (Wassum et al., 2009, 2011, 2016; Parkes and Balleine, 2013) but not $\mathrm{mOFC} \rightarrow \mathrm{BLA}$ projections (Malvaez et al., 2019). Thus, the $\mathrm{OOFC} \rightarrow$ BLA pathway mediates the formation of state-dependent reward memories, and the $\mathrm{mOFC} \rightarrow \mathrm{BLA}$ pathway facilitates the use of this information to guide adaptive reward-related behavior. Interestingly, however, under more dynamic decision scenarios the function of individual components of the OFC-BLA circuit can appear more overlapping. For example, during reversal learning, subjects must learn, integrate, and use information about reward availability and option value, and this form of decision making is influenced by lesion of $1 \mathrm{OFC} \rightarrow \mathrm{BLA}$ projections (Groman et al., 2019).

The $\mathrm{BLA} \rightarrow \mathrm{mOFC}$ pathway was found to mediate adaptive responses to cues based on the desirability of the predicted reward. This is consistent with prior evidence that the BLA is needed for sensitivity of Pavlovian responses to outcome-specific devaluation (Hatfield et al., 1996; Johnson et al., 2009) and evidence that BLA neuronal responses to reward-predictive cues can reflect the value of the predicted reward (Schoenbaum et al., 1998; Saddoris et al., 2005; Paton et al., 2006; Belova et al., 2007, 2008). The data here reveal this function to be achieved, at least in part, via projections to the $\mathrm{mOFC}$. $\mathrm{BLA} \rightarrow \mathrm{mOFC}$ projection activity was not necessary for reward cues to bias choice toward the predicted reward. Expression of such outcome-selective PIT depends on the sensory-specific identity of the predicted reward, but not its value (Holland, 2004). Thus, BLA $\rightarrow$ mOFC pathway activity mediates cue-driven behaviors based on a representation of the predicted reward's value but not its identity. Surprisingly, this indicates that a reward's identity can be decoupled from its value, that one can represent which specific reward is predicted but not the current value of that reward.
The function of the BLA $\rightarrow$ mOFC pathway identified here is distinct from that previously identified for the BLA $\rightarrow$ IOFC pathway. Using the same procedures, we previously found that activity in the anatomically distinct BLA $\rightarrow$ lOFC pathway is necessary for reward cues to both bias action choice toward the predicted reward and to adapt conditional approach responses based on the value of the predicted reward. Thus, the $\mathrm{BLA} \rightarrow$ IOFC pathway allows one to use cues to know which specific reward is predicted, whereas $\mathrm{BLA} \rightarrow \mathrm{mOFC}$ pathway activity promotes behavior based on the current desirability of that predicted reward. Whether BLA $\rightarrow$ IOFC function in value is secondary to representing reward identity (if you do not know which reward is predicted, then you cannot represent its value) is a critical open question.

Neither $\mathrm{mOFC} \rightarrow \mathrm{BLA}$ nor $\mathrm{BLA} \rightarrow \mathrm{mOFC}$ projections were necessary for general conditional goal-approach responses, consistent with evidence from BLA or mOFC lesions (Hatfield et al., 1996; Everitt et al., 2000; Parkinson et al., 2000; Corbit and Balleine, 2005; Bradfield et al., 2015, 2018; Malvaez et al., 2015; Morse et al., 2020). This well-learned cue response does not require a representation of the specific predicted reward or onthe-fly use of this information for prospective consideration of the most advantageous option. Instead, it can rely on a previously learned policy. Thus, mOFC-BLA circuitry is not needed for cue responses generally but is needed when one must use stimulus-outcome memories to infer what to do based on which outcomes are expected and the current desirability of the outcomes.

The mOFC-BLA circuit was also not needed for rats to adapt their instrumental choice behavior based on the value of the predicted reward. Rather, it is selectively needed for cues to inform the considerations guiding adaptive appetitive behavior. Both the BLA and $\mathrm{mOFC}$ are needed for the sensitivity of instrumental choice to devaluation (Balleine et al., 2003; Ostlund and Balleine, 2008; Johnson et al., 2009; Bradfield et al., 2015, 2018; Gourley et al., 2016). This function may, therefore, be achieved via alternate pathways, perhaps those to the striatum (Hoover and Vertes, 2011; Corbit et al., 2013; van Holstein et al., 2020), a region heavily implicated in action-outcome memory (Malvaez and Wassum, 2018; Malvaez et al., 2018b; Malvaez, 2020).

Collectively, these data reveal the mOFC-BLA circuit as critical for the cue-dependent reward outcome expectations that influence decision making and adaptive cue responses. mOFC neuronal activity can represent partially unobservable states (Lopatina et al., 2017; Elliott Wimmer and Büchel, 2019) and is needed when such states must be used to make adaptive choices (Bradfield et al., 2015, 2018). Activity in the $\mathrm{mOFC} \rightarrow$ BLA pathway may, therefore, be involved in signaling the state predicted by the reward cues. The BLA pathways to the IOFC and mOFC might then facilitate the use of this information to guide adaptive behavior, with BLA $\rightarrow$ IOFC projections facilitating detailed, sensory-specific representations of expected rewards (Lichtenberg et al., 2017) and BLA $\rightarrow$ mOFC projections facilitating the more general representations needed to compute option desirability. Indeed, lOFC neuronal activity can encode high-dimensional representations and the identity of predicted rewards (KleinFlügge et al., 2013; McDannald et al., 2014; Rudebeck and Murray, 2014; Wilson et al., 2014; Howard et al., 2015; Rich and Wallis, 2016; Suzuki et al., 2017; Rudebeck and Rich, 2018), whereas mOFC represents more general information about expected events that is used to make decisions based on value estimations or comparisons (Pritchard et al., 2005; PadoaSchioppa and Assad, 2006; Plassmann et al., 2010; Kennerley et 
al., 2011; Levy and Glimcher, 2011; Rudebeck and Murray, 2011; Burton et al., 2014; Lopatina et al., 2016, 2017; Suzuki et al., 2017). The precise information content conveyed by each component of the OFC-BLA circuit and how it is used in the receiving structure is a critical question for follow-up investigation. Because the staged scenarios used here allow isolation of psychological processes that typically co-occur and interact to influence decision making, such activity analysis will also be important for understanding how the functions identified here relate to more dynamic and complex decision-making scenarios. Another essential question is whether mOFC-BLA circuit function is similar in females, who do show similar performance in the tasks used here and require the BLA and $\mathrm{mOFC}$ for their performance (Ostlund and Balleine, 2008; Bradfield et al., 2018).

An inability to use reward cues to inform prospective considerations of which specific rewards can be expected and their current desirability can lead to maladaptive choices. Maladaptive choices could also arise if one is able to know what rewards are predicted, but not to consider their current value, as we showed here is neurobiologically possible. This is characteristic of the cognitive symptoms underlying many psychiatric diseases, including substance use disorder (Kalivas and Volkow, 2005; Verdejo-Garcia et al., 2018). Moreover, both the mOFC and BLA, as well as their connectivity, can be dysfunctional in substance use disorder and other psychiatric illnesses marked by disrupted appetitive decision making (Tanabe et al., 2009; Goldstein and Volkow, 2011; Hahn et al., 2011; Linke et al., 2012; Sladky et al., 2015; Shields and Gremel, 2020; Xie et al., 2021). Thus, these data may aid our understanding and treatment of these conditions.

\section{References}

Balleine BW, Dickinson A (1998) Goal-directed instrumental action: contingency and incentive learning and their cortical substrates. Neuropharmacology 37:407-419.

Balleine BW, Killcross AS, Dickinson A (2003) The effect of lesions of the basolateral amygdala on instrumental conditioning. J Neurosci 23:666-675.

Barreiros IV, Panayi MC, Walton ME (2021) Organization of afferents along the anterior-posterior and medial-lateral axes of the rat orbitofrontal cortex. Neuroscience 460:53-68.

Belova MA, Paton JJ, Morrison SE, Salzman CD (2007) Expectation modulates neural responses to pleasant and aversive stimuli in primate amygdala. Neuron 55:970-984.

Belova MA, Paton JJ, Salzman CD (2008) Moment-to-moment tracking of state value in the amygdala. J Neurosci 28:10023-10030.

Blundell P, Hall G, Killcross S (2001) Lesions of the basolateral amygdala disrupt selective aspects of reinforcer representation in rats. J Neurosci 21:9018-9026.

Bradfield LA, Dezfouli A, van Holstein M, Chieng B, Balleine BW (2015) Medial orbitofrontal cortex mediates outcome retrieval in partially observable task situations. Neuron 88:1268-1280.

Bradfield LA, Hart G, Balleine BW (2018) Inferring action-dependent outcome representations depends on anterior but not posterior medial orbitofrontal cortex. Neurobiol Learn Mem 155:463-473.

Bray S, Shimojo S, O'Doherty JP (2010) Human medial orbitofrontal cortex is recruited during experience of imagined and real rewards. J Neurophysiol 103:2506-2512.

Burton AC, Kashtelyan V, Bryden DW, Roesch MR (2014) Increased firing to cues that predict low-value reward in the medial orbitofrontal cortex. Cereb Cortex 24:3310-3321.

Collins AL, Aitken TJ, Huang IW, Shieh C, Greenfield VY, Monbouquette HG, Ostlund SB, Wassum KM (2019) Nucleus accumbens cholinergic interneurons oppose cue-motivated behavior. Biol Psychiatry 86:388396.

Colwill RM, Motzkin DK (1994) Encoding of the unconditioned stimulus in pavlovian conditioning. Animal Learning and Behavior 22:384-394.
Corbit LH, Balleine BW (2005) Double dissociation of basolateral and central amygdala lesions on the general and outcome-specific forms of pavlovian-instrumental transfer. J Neurosci 25:962-970.

Corbit LH, Balleine BW (2016) Learning and motivational processes contributing to pavlovian-instrumental transfer and their neural bases: dopamine and beyond. Curr Top Behav Neurosci 27:259-289.

Corbit LH, Leung BK, Balleine BW (2013) The role of the amygdala-striatal pathway in the acquisition and performance of goal-directed instrumental actions. J Neurosci 33:17682-17690.

Dalton GL, Wang NY, Phillips AG, Floresco SB (2016) Multifaceted contributions by different regions of the orbitofrontal and medial prefrontal cortex to probabilistic reversal learning. J Neurosci 36:1996-2006.

Delamater AR (2012) On the nature of CS and US representations in pavlovian learning. Learn Behav 40:1-23.

Elliott Wimmer G, Büchel C (2019) Learning of distant state predictions by the orbitofrontal cortex in humans. Nat Commun 10:2554.

Everitt BJ, Cardinal RN, Hall J, Parkinson JA, Robbins TW (2000) Differential involvement of amygdala subsystems in appetitive conditioning and drug addiction. In: The amygdala: A functional analysis (Aggleton JP, ed), pp 353-390. Oxford, England: Oxford UP.

Fanselow MS, Wassum KM (2015) The origins and organization of vertebrate pavlovian conditioning. Cold Spring Harb Perspect Biol 8:a021717.

Gallagher M, McMahan RW, Schoenbaum G (1999) Orbitofrontal cortex and representation of incentive value in associative learning. J Neurosci 19:6610-6614.

Gilroy KE, Everett EM, Delamater AR (2014) Response-outcome versus outcome-response associations in pavlovian-to-instrumental transfer: effects of instrumental training context. Int J Comp Psychol 27:585-597.

Goldstein RZ, Volkow ND (2011) Dysfunction of the prefrontal cortex in addiction: neuroimaging findings and clinical implications. Nat Rev Neurosci 12:652-669.

Gourley SL, Zimmermann KS, Allen AG, Taylor JR (2016) The medial orbitofrontal cortex regulates sensitivity to outcome value. J Neurosci 36:4600-4613.

Groman SM, Keistler C, Keip AJ, Hammarlund E, DiLeone RJ, Pittenger C, Lee D, Taylor JR (2019) Orbitofrontal circuits control multiple reinforcement-learning processes. Neuron 103:734-746.e3.

Hahn A, Stein P, Windischberger C, Weissenbacher A, Spindelegger C, Moser E, Kasper S, Lanzenberger R (2011) Reduced resting-state functional connectivity between amygdala and orbitofrontal cortex in social anxiety disorder. Neuroimage 56:881-889.

Hatfield T, Han JS, Conley M, Gallagher M, Holland P (1996) Neurotoxic lesions of basolateral, but not central, amygdala interfere with pavlovian second-order conditioning and reinforcer devaluation effects. J Neurosci 16:5256-5265.

Heilbronner SR, Rodriguez-Romaguera J, Quirk GJ, Groenewegen HJ, Haber SN (2016) Circuit-based corticostriatal homologies between rat and primate. Biol Psychiatry 80:509-521.

Holland PC (2004) Relations between pavlovian-instrumental transfer and reinforcer devaluation. J Exp Psychol Anim Behav Process 30:104-117.

Hoover WB, Vertes RP (2011) Projections of the medial orbital and ventral orbital cortex in the rat. J Comp Neurol 519:3766-3801.

Howard JD, Gottfried JA, Tobler PN, Kahnt T (2015) Identity-specific coding of future rewards in the human orbitofrontal cortex. Proc Natl Acad Sci U S A 112:5195-5200.

Izquierdo A (2017) Functional Heterogeneity within Rat Orbitofrontal Cortex in Reward Learning and Decision Making. J Neurosci 37:1052910540 .

Izquierdo A, Suda RK, Murray EA (2004) Bilateral orbital prefrontal cortex lesions in rhesus monkeys disrupt choices guided by both reward value and reward contingency. J Neurosci 24:7540-7548.

Johnson AW, Gallagher M, Holland PC (2009) The basolateral amygdala is critical to the expression of pavlovian and instrumental outcome-specific reinforcer devaluation effects. J Neurosci 29:696-704.

Kalivas PW, Volkow ND (2005) The neural basis of addiction: a pathology of motivation and choice. Am J Psychiatry 162:1403-1413.

Kennerley SW, Behrens TE, Wallis JD (2011) Double dissociation of value computations in orbitofrontal and anterior cingulate neurons. Nat Neurosci 14:1581-1589.

Kita H, Kitai ST (1990) Amygdaloid projections to the frontal cortex and the striatum in the rat. J Comp Neurol 298:40-49. 
Klein-Flügge MC, Barron HC, Brodersen KH, Dolan RJ, Behrens TE (2013) Segregated encoding of reward-identity and stimulus-reward associations in human orbitofrontal cortex. J Neurosci 33:3202-3211.

Kruse H, Overmier J, Konz W, Rokke E (1983) Pavlovian conditioned stimulus effects upon instrumental choice behavior are reinforcer specific. Learn Motiv 14:165-181.

Levy DJ, Glimcher PW (2011) Comparing apples and oranges: using rewardspecific and reward-general subjective value representation in the brain. J Neurosci 31:14693-14707.

Lichtenberg NT, Wassum KM (2017) Amygdala mu-opioid receptors mediate the motivating influence of cue-triggered reward expectations. Eur J Neurosci 45:381-387.

Lichtenberg NT, Pennington ZT, Holley SM, Greenfield VY, Cepeda C, Levine MS, Wassum KM (2017) Basolateral amygdala to orbitofrontal cortex projections enable cue-triggered reward expectations. J Neurosci $37: 8374-8384$

Linke J, King AV, Rietschel M, Strohmaier J, Hennerici M, Gass A, MeyerLindenberg A, Wessa M (2012) Increased medial orbitofrontal and amygdala activation: evidence for a systems-level endophenotype of bipolar I disorder. Am J Psychiatry 169:316-325.

Lopatina N, McDannald MA, Styer CV, Peterson JF, Sadacca BF, Cheer JF, Schoenbaum G (2016) Medial orbitofrontal neurons preferentially signal cues predicting changes in reward during unblocking. J Neurosci 36:8416-8424.

Lopatina N, Sadacca BF, McDannald MA, Styer CV, Peterson JF, Cheer JF, Schoenbaum G (2017) Ensembles in medial and lateral orbitofrontal cortex construct cognitive maps emphasizing different features of the behavioral landscape. Behav Neurosci 131:201-212.

Machado CJ, Bachevalier J (2007) The effects of selective amygdala, orbital frontal cortex or hippocampal formation lesions on reward assessment in nonhuman primates. Eur J Neurosci 25:2885-2904.

Malvaez M (2020) Neural substrates of habit. J Neurosci Res 98:986-997.

Malvaez M, Wassum K (2018) Regulation of habit formation in the dorsal striatum. Curr Opin Behav Sci 20:67-74.

Malvaez M, Greenfield VY, Wang AS, Yorita AM, Feng L, Linker KE, Monbouquette HG, Wassum KM (2015) Basolateral amygdala rapid glutamate release encodes an outcome-specific representation vital for reward-predictive cues to selectively invigorate reward-seeking actions. Sci Rep 5:12511.

Malvaez M, Shieh C, Murphy M, Greenfield V, Wassum K (2018a) Distinct cortical-amygdala projections drive reward value encoding and retrieval. BioRxiv 299958.

Malvaez M, Greenfield VY, Matheos DP, Angelillis NA, Murphy MD, Kennedy PJ, Wood MA, Wassum KM (2018b) Habits are negatively regulated by histone deacetylase 3 in the dorsal striatum. Biol Psychiatry 84:383-392.

Malvaez M, Shieh C, Murphy MD, Greenfield VY, Wassum KM (2019) Distinct cortical-amygdala projections drive reward value encoding and retrieval. Nat Neurosci 22:762-769.

McDannald MA, Esber GR, Wegener MA, Wied HM, Liu TL, Stalnaker TA, Jones JL, Trageser J, Schoenbaum G (2014) Orbitofrontal neurons acquire responses to "valueless" pavlovian cues during unblocking. Elife 3: e02653.

Morecraft RJ, Geula C, Mesulam MM (1992) Cytoarchitecture and neural afferents of orbitofrontal cortex in the brain of the monkey. J Comp Neurol 323:341-358.

Morse AK, Leung BK, Heath E, Bertran-Gonzalez J, Pepin E, Chieng BC, Balleine BW, Laurent V (2020) Basolateral amygdala drives a GPCRmediated striatal memory necessary for predictive learning to influence choice. Neuron 106:855-869.e8.

Münster A, Hauber W (2018) Medial orbitofrontal cortex mediates effortrelated responding in rats. Cereb Cortex 28:4379-4389.

Namboodiri VMK, Otis JM, van Heeswijk K, Voets ES, Alghorazi RA, Rodriguez-Romaguera J, Mihalas S, Stuber GD (2019) Single-cell activity tracking reveals that orbitofrontal neurons acquire and maintain a longterm memory to guide behavioral adaptation. Nat Neurosci 22:11101121.

Noonan MP, Walton ME, Behrens TE, Sallet J, Buckley MJ, Rushworth MF (2010) Separate value comparison and learning mechanisms in macaque medial and lateral orbitofrontal cortex. Proc Natl Acad Sci U S A 107:20547-20552.
Noonan MP, Chau B, Rushworth MF, Fellows LK (2017) Contrasting effects of medial and lateral orbitofrontal cortex lesions on credit assignment and decision making in humans. J Neurosci 37:7023-7035.

Ostlund SB, Balleine BW (2007) Orbitofrontal cortex mediates outcome encoding in pavlovian but not instrumental conditioning. J Neurosci 27:4819-4825.

Ostlund SB, Balleine BW (2008) Differential involvement of the basolateral amygdala and mediodorsal thalamus in instrumental action selection. J Neurosci 28:4398-4405.

Padoa-Schioppa C, Assad JA (2006) Neurons in the orbitofrontal cortex encode economic value. Nature 441:223-226.

Panayi MC, Killcross S (2018) Functional heterogeneity within the rodent lateral orbitofrontal cortex dissociates outcome devaluation and reversal learning deficits. Elife 7:e37357.

Parkes SL, Balleine BW (2013) Incentive memory: evidence the basolateral amygdala encodes and the insular cortex retrieves outcome values to guide choice between goal-directed actions. J Neurosci 33:8753-8763.

Parkinson JA, Robbins TW, Everitt BJ (2000) Dissociable roles of the central and basolateral amygdala in appetitive emotional learning. Eur J Neurosci 12:405-413.

Paton JJ, Belova MA, Morrison SE, Salzman CD (2006) The primate amygdala represents the positive and negative value of visual stimuli during learning. Nature 439:865-870.

Pickens CL, Saddoris MP, Setlow B, Gallagher M, Holland PC, Schoenbaum G (2003) Different roles for orbitofrontal cortex and basolateral amygdala in a reinforcer devaluation task. J Neurosci 23:11078-11084.

Pickens CL, Saddoris MP, Gallagher M, Holland PC (2005) Orbitofrontal lesions impair use of cue-outcome associations in a devaluation task. Behav Neurosci 119:317-322.

Plassmann H, O'Doherty JP, Rangel A (2010) Appetitive and aversive goal values are encoded in the medial orbitofrontal cortex at the time of decision making. J Neurosci 30:10799-10808.

Pritchard TC, Edwards EM, Smith CA, Hilgert KG, Gavlick AM, Maryniak TD, Schwartz GJ, Scott TR (2005) Gustatory neural responses in the medial orbitofrontal cortex of the old world monkey. J Neurosci 25:6047-6056

Reppucci CJ, Petrovich GD (2016) Organization of connections between the amygdala, medial prefrontal cortex, and lateral hypothalamus: a single and double retrograde tracing study in rats. Brain Struct Funct 221:29372962.

Rhodes SE, Murray EA (2013) Differential effects of amygdala, orbital prefrontal cortex, and prelimbic cortex lesions on goal-directed behavior in rhesus macaques. J Neurosci 33:3380-3389.

Rich EL, Wallis JD (2016) Decoding subjective decisions from orbitofrontal cortex. Nat Neurosci 19:973-980.

Rudebeck PH, Murray EA (2011) Balkanizing the primate orbitofrontal cortex: distinct subregions for comparing and contrasting values. Ann N Y Acad Sci 1239:1-13.

Rudebeck PH, Murray EA (2014) The orbitofrontal oracle: cortical mechanisms for the prediction and evaluation of specific behavioral outcomes. Neuron 84:1143-1156.

Rudebeck P, Rich E (2018) Orbitofrontal cortex. Curr Biol 28:R1083-R1088.

Saddoris MP, Gallagher M, Schoenbaum G (2005) Rapid associative encoding in basolateral amygdala depends on connections with orbitofrontal cortex. Neuron 46:321-331.

Schoenbaum G, Chiba AA, Gallagher M (1998) Orbitofrontal cortex and basolateral amygdala encode expected outcomes during learning. Nat Neurosci 1:155-159.

Shields CN, Gremel CM (2020) Review of orbitofrontal cortex in alcohol dependence: a disrupted cognitive map? Alcohol Clin Exp Res 44:19521964.

Sias A, Morse A, Wang S, Greenfield V, Goodpaster C, Wrenn T, Wikenheiser A, Holley S, Cepeda C, Levine M, Wassum K (2021) A bidirectional corticoamygdala circuit for the encoding and retrieval of detailed reward memories. eLife 10:e68617.

Sladky R, Höflich A, Küblböck M, Kraus C, Baldinger P, Moser E, Lanzenberger R, Windischberger C (2015) Disrupted effective connectivity between the amygdala and orbitofrontal cortex in social anxiety disorder during emotion discrimination revealed by dynamic causal modeling for FMRI. Cereb Cortex 25:895-903. 
Stopper CM, Green EB, Floresco SB (2014) Selective involvement by the medial orbitofrontal cortex in biasing risky, but not impulsive, choice. Cereb Cortex 24:154-162.

Suzuki S, Cross L, O’Doherty JP (2017) Elucidating the underlying components of food valuation in the human orbitofrontal cortex. Nat Neurosci 20:1780-1786.

Tanabe J, Tregellas JR, Dalwani M, Thompson L, Owens E, Crowley T, Banich M (2009) Medial orbitofrontal cortex gray matter is reduced in abstinent substance-dependent individuals. Biol Psychiatry 65:160-164.

van Holstein M, MacLeod PE, Floresco SB (2020) Basolateral amygdala-nucleus accumbens circuitry regulates optimal cue-guided risk/reward decision making. Prog Neuropsychopharmacol Biol Psychiatry 98:109830.

Verdejo-Garcia A, Chong TT, Stout JC, Yücel M, London ED (2018) Stages of dysfunctional decision-making in addiction. Pharmacol Biochem Behav 164:99-105.

Wallis JD (2011) Cross-species studies of orbitofrontal cortex and valuebased decision-making. Nat Neurosci 15:13-19.

Wassum KM, Ostlund SB, Maidment NT, Balleine BW (2009) Distinct opioid circuits determine the palatability and the desirability of rewarding events. Proc Natl Acad Sci U S A 106:12512-12517.

Wassum KM, Cely IC, Balleine BW, Maidment NT (2011) $\mu$-Opioid receptor activation in the basolateral amygdala mediates the learning of increases but not decreases in the incentive value of a food reward. J Neurosci 31:1591- 1599 .

Wassum KM, Greenfield VY, Linker KE, Maidment NT, Ostlund SB (2016) Inflated reward value in early opiate withdrawal. Addict Biol 21:221-233.

Wellman LL, Gale K, Malkova L (2005) GABAA-mediated inhibition of basolateral amygdala blocks reward devaluation in macaques. J Neurosci 25:4577-4586.

West EA, DesJardin JT, Gale K, Malkova L (2011) Transient inactivation of orbitofrontal cortex blocks reinforcer devaluation in macaques. J Neurosci 31:15128-15135.

Wilson RC, Takahashi YK, Schoenbaum G, Niv Y (2014) Orbitofrontal cortex as a cognitive map of task space. Neuron 81:267-279.

Woon EP, Sequeira MK, Barbee BR, Gourley SL (2020) Involvement of the rodent prelimbic and medial orbitofrontal cortices in goal-directed action: a brief review. J Neurosci Res 98:1020-1030.

Xie C, Jia T, Rolls ET, Robbins TW, Sahakian BJ, Zhang J, Liu Z, Cheng W, Luo Q, Zac Lo C-Y, Wang H, Banaschewski T, Barker GJ, Bokde ALW, Büchel C, Quinlan EB, Desrivières S, Flor H, Grigis A, Garavan H, et al. (2021) Reward versus nonreward sensitivity of the medial versus lateral orbitofrontal cortex relates to the severity of depressive symptoms. Biol Psychiatry Cogn Neurosci Neuroimaging 6:259-269.

Yamada H, Louie K, Tymula A, Glimcher PW (2018) Free choice shapes normalized value signals in medial orbitofrontal cortex. Nat Commun 9:162. 